$\mathrm{J}$ o u r n a l of

Mathematics

and Applications

JMA No 42, pp 35-61 (2019)

\title{
Existence and Uniqueness of Solutions for Nonlinear Katugampola Fractional Differential Equations
}

\author{
Bilal Basti, Yacine Arioua* and Nouredine Benhamidouche
}

\begin{abstract}
The present paper deals with the existence and uniqueness of solutions for a boundary value problem of nonlinear fractional differential equations with Katugampola fractional derivative. The main results are proved by means of Guo-Krasnoselskii and Banach fixed point theorems. For applications purposes, some examples are provided to demonstrate the usefulness of our main results.
\end{abstract}

AMS Subject Classification: 34A08, 34A37.

Keywords and Phrases: Fractional equation; Fixed point theorems; Boundary value problem; Existence; Uniqueness.

\section{Introduction}

The differential equations of fractional order are generalizations of classical differential equations of integer order. They are increasingly used in a variety of fields such as fluid flow, control theory of dynamical systems, signal and image processing, aerodynamics, electromagnetics, probability and statistics, (Samko et al. 1993 [18], Podlubny 1999 [17], Kilbas et al. 2006 [9], Diethelm 2010 [3]) books can be checked as a reference.

Boundary value problem of fractional differential equations is recently approached by various researchers ([1], [8], [19], [20]).

In [20], Bai and L used some fixed point theorems on cone to show the existence and multiplicity of positive solutions for a Dirichlet-type problem of the nonlinear fractional differential equation:

$$
\left\{\begin{array}{l}
\mathcal{D}_{0^{+}}^{\alpha} u(t)+f(t, u(t))=0, \quad 0<t<1, \\
u(0)=u(1)=0,
\end{array}\right.
$$


where $\mathcal{D}_{0^{+}}^{\alpha} u$ is the standard Riemann Liouville fractional derivative of order $1<\alpha \leq 2$ and $f:[0,1] \times[0, \infty) \rightarrow[0, \infty)$ is continuous function.

In a recent work [8], Katugampola studied the existence and uniqueness of solutions for the following initial value problem:

$$
\left\{\begin{array}{l}
{ }_{c}^{\rho} \mathcal{D}_{0^{+}}^{\alpha} u(t)=f(t, u(t)), \alpha>0, \\
D^{k} u(0)=u_{0}^{(k)}, k=1,2, \ldots, m-1,
\end{array}\right.
$$

where $m=[\alpha],{ }_{c}^{\rho} \mathcal{D}_{0^{+}}^{\alpha}$ is the Caputo-type generalized fractional derivative, of order $\alpha$, and $f: G \rightarrow \mathbb{R}$ is a given continuous function with:

$$
G=\left\{(t, u): t \in\left[0, h^{*}\right],\left|u-\sum_{k=0}^{m-1} \frac{t^{k} u_{0}^{(k)}}{k !}\right| \leq K, K, h^{*}>0\right\} .
$$

This paper focuses on the existence and uniqueness of solutions for a nonlinear fractional differential equation involving Katugampola fractional derivative:

$$
{ }^{\rho} \mathcal{D}_{0^{+}}^{\alpha} u(t)+\beta f(t, u(t))=0,0<t<T,
$$

supplemented with the boundary conditions:

$$
u(0)=0, u(T)=0,
$$

where $\beta \in \mathbb{R}$, and ${ }^{\rho} \mathcal{D}_{0^{+}}^{\alpha}$ for $\rho>0$, presents Katugampola fractional derivative of order $1<\alpha \leq 2, f:[0, T] \times[0, \infty) \rightarrow[h, \infty)$ is a continuous function, with finite positive constants $h, T$.

\section{Background materials and preliminaries}

In this section, some necessary definitions from fractional calculus theory are presented. Let $\Omega=[0, T] \subset \mathbb{R}$ be a finite interval.

As in [9], let us denote by $X_{c}^{p}[0, T],(c \in \mathbb{R}, 1 \leq p \leq \infty)$ the space of those complex-valued Lebesgue measurable functions $y$ on $[0, T]$ for which $\|y\|_{X_{c}^{p}}<\infty$ is defined by

$$
\|y\|_{X_{c}^{p}}=\left(\int_{0}^{T}\left|s^{c} y(s)\right|^{p} \frac{d s}{s}\right)^{\frac{1}{p}}<\infty
$$

for $1 \leq p<\infty, c \in \mathbb{R}$, and

$$
\|y\|_{X_{c}^{\infty}}=\operatorname{ess} \sup _{0 \leq t \leq T}\left[t^{c}|y(t)|\right],(c \in \mathbb{R}) .
$$

Definition 2.1 (Riemann-Liouville fractional integral [9]). The left-sided RiemannLiouville fractional integral of order $\alpha>0$ of a continuous function $y:[0, T] \rightarrow \mathbb{R}$ is given by:

$$
{ }^{R L} \mathcal{I}_{0^{+}}^{\alpha} y(t)=\frac{1}{\Gamma(\alpha)} \int_{0}^{t}(t-s)^{\alpha-1} y(s) d s, t \in[0, T],
$$

where $\Gamma(\alpha)=\int_{0}^{+\infty} e^{-s} s^{\alpha-1} d s$, is the Euler gamma function. 
Definition 2.2 (Riemann-Liouville fractional derivative [9]). The left-sided Riemann Liouville fractional derivative of order $\alpha>0$ of a continuous function $y:[0, T] \rightarrow \mathbb{R}$ is given by:

$$
R L \mathcal{D}_{0^{+}}^{\alpha} y(t)=\frac{1}{\Gamma(n-\alpha)}\left(\frac{d}{d t}\right)^{n} \int_{0}^{t}(t-s)^{n-\alpha-1} y(s) d s, t \in[0, T], n=[\alpha]+1
$$

Definition 2.3 (Hadamard fractional integral [9]). The left-sided Hadamard fractional integral of order $\alpha>0$ of a continuous function $y:[0, T] \rightarrow \mathbb{R}$ is given by:

$$
{ }^{H} \mathcal{I}_{0^{+}}^{\alpha} y(t)=\frac{1}{\Gamma(\alpha)} \int_{0}^{t}\left(\log \frac{t}{s}\right){ }^{\alpha-1} y(s) \frac{d s}{s}, \quad t \in[0, T]
$$

Definition 2.4 (Hadamard fractional derivative [9]). The left-sided Hadamard fractional derivative of order $\alpha>0$ of a continuous function $y:[0, T] \rightarrow \mathbb{R}$ is given by:

$$
{ }^{H} \mathcal{D}_{0^{+}}^{\alpha} y(t)=\frac{1}{\Gamma(n-\alpha)}\left(t \frac{d}{d t}\right)^{n} \int_{0}^{t}\left(\log \frac{t}{s}\right)^{n-\alpha-1} y(s) \frac{d s}{s}, \quad t \in[0, T], n=[\alpha]+1
$$

if the integral exist.

A recent generalization in 2011, introduced by Udita Katugampola [6], combines the Riemann-Liouville fractional integral and the Hadamard fractional integral into a single form (see [9]), the integral is now known as Katugampola fractional integral, it is given in the following definition:

Definition 2.5 (Katugampola fractional integral [6]).

The left-sided Katugampola fractional integral of order $\alpha>0$ of a function $y \in$ $X_{c}^{p}[0, T]$ is defined by:

$$
\left({ }^{\rho} \mathcal{I}_{0^{+}}^{\alpha} y\right)(t)=\frac{\rho^{1-\alpha}}{\Gamma(\alpha)} \int_{0}^{t} \frac{s^{\rho-1} y(s)}{\left(t^{\rho}-s^{\rho}\right)^{1-\alpha}} d s, \rho>0, t \in[0, T]
$$

Similarly, we can define right-sided integrals [6]-[7], [9].

Definition 2.6 (Katugampola fractional derivatives [7]).

Let $\alpha, \rho \in \mathbb{R}^{+}$, and $n=[\alpha]+1$. The Katugampola fractional derivative corresponding to the Katugampola fractional integral (2.1) are defined for $0 \leq t \leq T \leq \infty$ by:

$$
{ }^{\rho} \mathcal{D}_{0^{+}}^{\alpha} y(t)=\left(t^{1-\rho} \frac{d}{d t}\right)^{n}\left({ }^{\rho} \mathcal{I}_{0^{+}}^{n-\alpha} y\right)(t)=\frac{\rho^{\alpha-n+1}}{\Gamma(n-\alpha)}\left(t^{1-\rho} \frac{d}{d t}\right)^{n} \int_{0}^{t} \frac{s^{\rho-1} y(s)}{\left(t^{\rho}-s^{\rho}\right)^{\alpha-n+1}} d s
$$


Theorem $2.7([7])$. Let $\alpha, \rho \in \mathbb{R}^{+}$, then

$$
\begin{aligned}
\lim _{\rho \rightarrow 1}\left({ }^{\rho} \mathcal{I}_{0^{+}}^{\alpha} y\right)(t) & ={ }^{R L} \mathcal{I}_{0^{+}}^{\alpha} y(t)=\frac{1}{\Gamma(\alpha)} \int_{0}^{t}(t-s)^{\alpha-1} y(s) d s \\
\lim _{\rho \rightarrow 0^{+}}\left({ }^{\rho} \mathcal{I}_{0^{+}}^{\alpha} y\right)(t) & ={ }^{H} \mathcal{I}_{0^{+}}^{\alpha} y(t)=\frac{1}{\Gamma(\alpha)} \int_{0}^{t}\left(\log \frac{t}{s}\right)^{\alpha-1} \frac{y(s)}{s} d s \\
\lim _{\rho \rightarrow 1}\left({ }^{\rho} \mathcal{D}_{0^{+}}^{\alpha} y\right)(t) & ={ }^{R L} \mathcal{D}_{0^{+}}^{\alpha} y(t)=\frac{1}{\Gamma(n-\alpha)}\left(\frac{d}{d t}\right)^{n} \int_{0}^{t}(t-s)^{n-\alpha-1} y(s) d s \\
\lim _{\rho \rightarrow 0^{+}}\left({ }^{\rho} \mathcal{D}_{0^{+}}^{\alpha} y\right)(t) & ={ }^{H} \mathcal{D}_{0^{+}}^{\alpha} y(t)=\frac{1}{\Gamma(n-\alpha)}\left(t \frac{d}{d t}\right)^{n} \int_{0}^{t}\left(\log \frac{t}{s}\right)^{n-\alpha-1} \frac{y(s)}{s} d s .
\end{aligned}
$$

Remark. As an example, for $\alpha, \rho>0$, and $\mu>-\rho$, we have

$$
{ }^{\rho} \mathcal{D}_{0^{+}}^{\alpha} t^{\mu}=\frac{\rho^{\alpha-1} \Gamma\left(1+\frac{\mu}{\rho}\right)}{\Gamma\left(1-\alpha+\frac{\mu}{\rho}\right)} t^{\mu-\alpha \rho}
$$

In particular

$$
{ }^{\rho} \mathcal{D}_{0^{+}}^{\alpha} t^{\rho(\alpha-m)}=0 \text {, for each } m=1,2, \ldots, n .
$$

For $\mu>-\rho$, we have

$$
\begin{aligned}
{ }^{\rho} \mathcal{D}_{0^{+}}^{\alpha} t^{\mu} & =\frac{\rho^{\alpha-n+1}}{\Gamma(n-\alpha)}\left(t^{1-\rho} \frac{d}{d t}\right)^{n} \int_{0}^{t} s^{\rho+\mu-1}\left(t^{\rho}-s^{\rho}\right)^{n-\alpha-1} d s \\
& =\frac{\rho^{\alpha-n}}{\Gamma(n-\alpha)}\left(t^{1-\rho} \frac{d}{d t}\right)^{n} t^{\rho(n-\alpha)+\mu} \int_{0}^{1} \tau^{\frac{\mu}{\rho}}(1-\tau)^{n-\alpha-1} d \tau \\
& =\frac{\rho^{\alpha-n}}{\Gamma(n-\alpha)} B\left(n-\alpha, 1+\frac{\mu}{\rho}\right)\left(t^{1-\rho} \frac{d}{d t}\right)^{n} t^{\rho(n-\alpha)+\mu} \\
& =\frac{\rho^{\alpha-n} \Gamma\left(1+\frac{\mu}{\rho}\right)}{\Gamma\left(1+n-\alpha+\frac{\mu}{\rho}\right)}\left(t^{1-\rho} \frac{d}{d t}\right)^{n} t^{\rho(n-\alpha)+\mu} .
\end{aligned}
$$

Then

$$
{ }^{\rho} \mathcal{D}_{0^{+}}^{\alpha} t^{\mu}=\frac{\rho^{\alpha-1} \Gamma\left(1+\frac{\mu}{\rho}\right)}{\Gamma\left(1+n-\alpha+\frac{\mu}{\rho}\right)}\left[n-\alpha+\frac{\mu}{\rho}\right]\left[n-\alpha-1+\frac{\mu}{\rho}\right] \cdots\left[1-\alpha+\frac{\mu}{\rho}\right] t^{\mu-\alpha \rho} .
$$

As

$\Gamma\left(1+n-\alpha+\frac{\mu}{\rho}\right)=\left[n-\alpha+\frac{\mu}{\rho}\right]\left[n-\alpha-1+\frac{\mu}{\rho}\right] \ldots\left[1-\alpha+\frac{\mu}{\rho}\right] \Gamma\left(1-\alpha+\frac{\mu}{\rho}\right)$,

we get

$$
{ }^{\rho} \mathcal{D}_{0^{+}}^{\alpha} t^{\mu}=\frac{\rho^{\alpha-1} \Gamma\left(1+\frac{\mu}{\rho}\right)}{\Gamma\left(1-\alpha+\frac{\mu}{\rho}\right)} t^{\mu-\alpha \rho}
$$


In case $m=\alpha-\frac{\mu}{\rho}$, it follows from (2.4), that

$$
{ }^{\rho} \mathcal{D}_{0^{+}}^{\alpha} t^{\rho(\alpha-m)}=\rho^{\alpha-1} \frac{\Gamma(\alpha-m+1)}{\Gamma(n-m+1)}(n-m)(n-m-1) \cdots(1-m) t^{-\rho m} .
$$

So, for $m=1,2, \ldots, n$, we get

$$
{ }^{\rho} \mathcal{D}_{0^{+}}^{\alpha} t^{\rho(\alpha-m)}=0
$$

Similarly, for all $\alpha, \rho>0$, we have:

$$
{ }^{\rho} \mathcal{I}_{0^{+}}^{\alpha} t^{\mu}=\frac{\rho^{-\alpha} \Gamma\left(1+\frac{\mu}{\rho}\right)}{\Gamma\left(1+\alpha+\frac{\mu}{\rho}\right)} t^{\mu+\alpha \rho}, \forall \mu>-\rho .
$$

By $C[0, T]$, we denote the Banach space of all continuous functions from $[0, T]$ into $\mathbb{R}$ with the norm:

$$
\|y\|=\max _{0 \leq t \leq T}|y(t)|
$$

Remark. Let $p \geq 1, c>0$ and $T \leq(p c)^{\frac{1}{p c}}$. Far all $y \in C[0, T]$, note that

$$
\|y\|_{X_{c}^{p}}=\left(\int_{0}^{T}\left|s^{c} y(s)\right|^{p} \frac{d s}{s}\right)^{\frac{1}{p}} \leq\left(\|y\|^{p} \int_{0}^{T} s^{p c-1} d s\right)^{\frac{1}{p}}=\frac{T^{c}}{(p c)^{\frac{1}{p}}}\|y\|,
$$

and

$$
\|y\|_{X_{c}^{\infty}}=\operatorname{ess} \sup _{0 \leq t \leq T}\left[t^{c}|y(t)|\right] \leq T^{c}\|y\|,
$$

which imply that $C[0, T] \hookrightarrow X_{c}^{p}[0, T]$, and

$$
\|y\|_{X_{c}^{p}} \leq\|y\|_{\infty}, \text { for all } T \leq(p c)^{\frac{1}{p c}} .
$$

We express some properties of Katugampola fractional integral and derivative in the following result.

Theorem 2.8 ([6]-[7]-[8]).

Let $\alpha, \beta, \rho, c \in \mathbb{R}$, be such that $\alpha, \beta, \rho>0$. Then, for any $y \in X_{c}^{p}[0, T]$, where $1 \leq p \leq \infty$, we have:

- Index property:

$$
\begin{array}{ll}
{ }^{\rho} \mathcal{I}_{0^{+}}^{\alpha}{ }^{\rho} \mathcal{I}_{0^{+}}^{\beta} y(t) & ={ }^{\rho} \mathcal{I}_{0^{+}}^{\alpha+\beta} y(t),
\end{array}
$$

- Inverse property

$$
{ }^{\rho} \mathcal{D}_{0^{+}}^{\alpha}{ }^{\rho} \mathcal{I}_{0^{+}}^{\alpha} y(t)=y(t), \text { for all } \alpha \in(0,1) .
$$


From Definitions 2.5 and 2.6, and Theorem 2.8, we deduce that

$$
\begin{aligned}
{ }^{\rho} \mathcal{I}_{0^{+}}^{1}\left(t^{1-\rho} \frac{d}{d t}\right){ }^{\rho} \mathcal{I}_{0^{+}}^{\alpha+1} y(t) & =\int_{0}^{t} s^{\rho-1}\left(s^{1-\rho} \frac{d}{d s}\right){ }^{\rho} \mathcal{I}_{0^{+}}^{\alpha+1} y(s) d s \\
& =\int_{0}^{t} \frac{d}{d s}{ }^{\rho} \mathcal{I}_{0^{+}}^{\alpha+1} y(s) d s \\
& =\left[\frac{1}{\rho^{\alpha} \Gamma(\alpha+1)} \int_{0}^{s} \tau^{\rho-1}\left(t^{\rho}-\tau^{\rho}\right)^{\alpha} y(\tau) d \tau\right]_{0}^{t} \\
& ={ }^{\rho} \mathcal{I}_{0^{+}}^{\alpha+1} y(t) .
\end{aligned}
$$

Consequently

$$
\left(t^{1-\rho} \frac{d}{d t}\right){ }^{\rho} \mathcal{I}_{0^{+}}^{\alpha+1} y(t)={ }^{\rho} \mathcal{I}_{0^{+}}^{\alpha} y(t), \forall \alpha>0 .
$$

Definition 2.9 ([4]). Let $E$ be a real Banach space, a nonempty closed convex set $P \subset E$ is called a cone of $E$ if it satisfies the following conditions:

(i) $u \in P, \lambda \geq 0$, implies $\lambda u \in P$.

(ii) $u \in P,-u \in P$, implies $u=0$.

Definition 2.10 ([2]). Let $E$ be a Banach space, $P \in C(E)$ is called an equicontinuous part if and only if

$$
\forall \varepsilon>0, \exists \delta>0, \forall u, v \in E, \forall \mathcal{A} \in P,\|u-v\|<\delta \Rightarrow\|\mathcal{A}(u)-\mathcal{A}(v)\|<\varepsilon .
$$

Theorem 2.11 (Ascoli-Arzel [2]). Let $E$ be a compact space. If $\mathcal{A}$ is an equicontinuous, bounded subset of $C(E)$, then $\mathcal{A}$ is relatively compact.

Definition 2.12 (Completely continuous [4]). We say $\mathcal{A}: E \rightarrow E$ is completely continuous if for any bounded subset $P \subset E$, the set $\mathcal{A}(P)$ is relatively compact.

The following fixed-point theorems are fundamental in the proofs of our main results.

Lemma 2.13 (Guo-Krasnosel'skii fixed point theorems [12]).

Let $E$ be a Banach space, $P \subseteq E$ a cone, and $\Omega_{1}, \Omega_{2}$ two bounded open balls of $E$ centered at the origin with $\overline{\bar{\Omega}}_{1} \subset \Omega_{2}$. Suppose that $\mathcal{A}: P \cap\left(\bar{\Omega}_{2} \backslash \Omega_{1}\right) \rightarrow P$ is a completely continuous operator such that either

(i) $\|\mathcal{A} x\| \leq\|x\|, x \in P \cap \partial \Omega_{1}$ and $\|\mathcal{A} x\| \geq\|x\|, x \in P \cap \partial \Omega_{2}$, or

(ii) $\|\mathcal{A} x\| \geq\|x\|, x \in P \cap \partial \Omega_{1}$ and $\|\mathcal{A} x\| \leq\|x\|, x \in P \cap \partial \Omega_{2}$,

holds. Then $\mathcal{A}$ has a fixed point in $P \cap\left(\bar{\Omega}_{2} \backslash \Omega_{1}\right)$.

Theorem 2.14 (Banach's fixed point [5]). Let $E$ be a Banach space, $P \subseteq E$ a nonempty closed subset. If $\mathcal{A}: P \rightarrow P$ is a contraction mapping, then $\mathcal{A}$ has a unique fixed point in $P$. 


\section{Main results}

In the sequel, $T, p$ and $c$ are real constants such that

$$
p \geq 1, c>0, \text { and } T \leq(p c)^{\frac{1}{p c}} .
$$

Now, we present some important lemmas which play a key role in the proofs of the main results.

Lemma 3.1. Let $\alpha, \rho \in \mathbb{R}^{+}$. If $u \in C[0, T]$, then:

(i) The fractional equation ${ }^{\rho} \mathcal{D}_{0^{+}}^{\alpha} u(t)=0$, has a solution as follows:

$u(t)=C_{1} t^{\rho(\alpha-1)}+C_{2} t^{\rho(\alpha-2)}+\cdots+C_{n} t^{\rho(\alpha-n)}$, where $C_{m} \in \mathbb{R}$, with $m=1,2, \ldots, n$.

(ii) If ${ }^{\rho} \mathcal{D}_{0^{+}}^{\alpha} u \in C[0, T]$ and $1<\alpha \leq 2$, then:

$$
{ }^{\rho} \mathcal{I}_{0^{+}}^{\alpha}{ }^{\rho} \mathcal{D}_{0^{+}}^{\alpha} u(t)=u(t)+C_{1} t^{\rho(\alpha-1)}+C_{2} t^{\rho(\alpha-2)}, \text { for some } C_{1}, C_{2} \in \mathbb{R} .
$$

Proof. (i) Let $\alpha, \rho \in \mathbb{R}^{+}$. From remark 2, we have:

$$
{ }^{\rho} \mathcal{D}_{0^{+}}^{\alpha} t^{\rho(\alpha-m)}=0, \text { for each } m=1,2, \ldots, n .
$$

Then, the fractional differential equation ${ }^{\rho} \mathcal{D}_{0^{+}}^{\alpha} u(t)=0$, admits a solution as follows:

$$
u(t)=C_{1} t^{\rho(\alpha-1)}+C_{2} t^{\rho(\alpha-2)}+\cdots+C_{n} t^{\rho(\alpha-n)}, C_{m} \in \mathbb{R}, m=1,2, \ldots, n .
$$

(ii) Let ${ }^{\rho} \mathcal{D}_{0^{+}}^{\alpha} u \in C[0, T]$ be the fractional derivative (2.2) of order $1<\alpha \leq 2$. If we apply the operator ${ }^{\rho} \mathcal{I}_{0^{+}}^{\alpha}$ to ${ }^{\rho} \mathcal{D}_{0^{+}}^{\alpha} u(t)$ and use Definitions 2.5, 2.6, Theorem 2.8 and property (2.6), we get

$$
\begin{aligned}
& { }^{\rho} \mathcal{I}_{0^{+}}^{\alpha}{ }^{\rho} \mathcal{D}_{0^{+}}^{\alpha} u(t)=\left(t^{1-\rho} \frac{d}{d t}\right){ }^{\rho} \mathcal{I}_{0^{+}}^{\alpha+1}{ }^{\rho} \mathcal{D}_{0^{+}}^{\alpha} u(t) \\
& =\left(t^{1-\rho} \frac{d}{d t}\right)\left[\frac{\rho^{-\alpha}}{\Gamma(\alpha+1)} \int_{0}^{t}\left(t^{\rho}-s^{\rho}\right)^{\alpha} s^{\rho-1} \rho \mathcal{D}_{0^{+}}^{\alpha} u(s) d s\right] \\
& =\left(t^{1-\rho} \frac{d}{d t}\right)\left[\frac{\rho^{-\alpha}}{\Gamma(\alpha+1)} \int_{0}^{t}\left(t^{\rho}-s^{\rho}\right)^{\alpha} s^{\rho-1}\left[\left(s^{1-\rho} \frac{d}{d s}\right)^{2}{ }^{2} \mathcal{I}_{0^{+}}^{2-\alpha} u(s)\right] d s\right] \\
& =\left(t^{1-\rho} \frac{d}{d t}\right)\left[\frac{\rho^{-\alpha}}{\Gamma(\alpha+1)} \int_{0}^{t}\left(t^{\rho}-s^{\rho}\right)^{\alpha} \frac{d}{d s}\left[\left(s^{1-\rho} \frac{d}{d s}\right){ }^{\rho} \mathcal{I}_{0^{+}}^{2-\alpha} u(s)\right] d s\right] \\
& =\left(t^{1-\rho} \frac{d}{d t}\right)\left[\frac { \rho ^ { - \alpha } } { \Gamma ( \alpha + 1 ) } \left(\left[\left(t^{\rho}-s^{\rho}\right)^{\alpha}\left(s^{1-\rho} \frac{d}{d s}\right){ }^{\rho} \mathcal{I}_{0^{+}}^{2-\alpha} u(s)\right]_{0}^{t}\right.\right. \\
& \left.\left.+\alpha \rho \int_{0}^{t} s^{\rho-1}\left(t^{\rho}-s^{\rho}\right)^{\alpha-1}\left(s^{1-\rho} \frac{d}{d s}\right){ }^{\rho} \mathcal{I}_{0^{+}}^{2-\alpha} u(s) d s\right)\right] \text {. }
\end{aligned}
$$


From (2.6), we have

$$
\left(s^{1-\rho} \frac{d}{d s}\right){ }^{\rho} \mathcal{I}_{0^{+}}^{2-\alpha} u(s)={ }^{\rho} \mathcal{I}_{0^{+}}^{1-\alpha} u(s) .
$$

On the other hand, from (2.2), we have

$$
\left(s^{1-\rho} \frac{d}{d s}\right){ }^{\rho} \mathcal{I}_{0^{+}}^{2-\alpha} u(s)=\left(s^{1-\rho} \frac{d}{d s}\right)^{1}{ }^{\rho} \mathcal{I}_{0^{+}}^{1-(\alpha-1)} u(s)={ }^{\rho} \mathcal{D}_{0^{+}}^{\alpha-1} u(s) .
$$

Then

$$
\begin{aligned}
{ }^{\rho} \mathcal{I}_{0^{+}}^{\alpha}{ }^{\rho} \mathcal{D}_{0^{+}}^{\alpha} u(t)= & \underbrace{t^{1-\rho} \frac{d}{d t}\left(\frac{\rho^{1-\alpha}}{\Gamma(\alpha)} \int_{0}^{t}\left(t^{\rho}-s^{\rho}\right)^{\alpha-1} \frac{d}{d s}{ }^{\rho} \mathcal{I}_{0^{+}}^{2-\alpha} u(s) d s\right)}_{\psi} \\
& -\frac{\rho^{1-\alpha} \rho \mathcal{I}_{0^{+}}^{1-\alpha} u\left(0^{+}\right)}{\Gamma(\alpha)} t^{\rho(\alpha-1)},
\end{aligned}
$$

where

$$
\begin{aligned}
& \psi=t^{1-\rho} \frac{d}{d t} \frac{\rho^{1-\alpha}}{\Gamma(\alpha)}\left(\left[\left(t^{\rho}-s^{\rho}\right)^{\alpha-1} \rho \mathcal{I}_{0^{+}}^{2-\alpha} u(s)\right]_{0}^{t}\right. \\
& \left.+\rho(\alpha-1) \int_{0}^{t} s^{\rho-1}\left(t^{\rho}-s^{\rho}\right)^{\alpha-2} \rho \mathcal{I}_{0^{+}}^{2-\alpha} u(s) d s\right) \\
& =t^{1-\rho} \frac{d}{d t}\left(\frac{\rho^{2-\alpha}}{\Gamma(\alpha-1)} \int_{0}^{t} s^{\rho-1}\left(t^{\rho}-s^{\rho}\right)^{\alpha-2} \rho \mathcal{I}_{0^{+}}^{2-\alpha} u(s) d s\right. \\
& \left.-\frac{\rho^{1-\alpha} \rho}{\mathcal{I}_{0^{+}}^{2-\alpha} u\left(0^{+}\right)} t^{\rho(\alpha-1)}\right) \\
& =t^{1-\rho} \frac{d}{d t}\left({ }^{\rho} \mathcal{I}_{0^{+}}^{\alpha-1}{ }^{\rho} \mathcal{I}_{0^{+}}^{2-\alpha} u(t)-\frac{\rho^{1-\alpha} \rho \mathcal{I}_{0^{+}}^{2-\alpha} u\left(0^{+}\right)}{\Gamma(\alpha)} t^{\rho(\alpha-1)}\right) \\
& =t^{1-\rho} \frac{d}{d t}\left({ }^{\rho} \mathcal{I}_{0^{+}}^{1} u(t)-\frac{\rho^{1-\alpha} \rho \mathcal{I}_{0^{+}}^{2-\alpha} u\left(0^{+}\right)}{\Gamma(\alpha)} t^{\rho(\alpha-1)}\right) \\
& =u(t)-\frac{\rho^{2-\alpha}{ }^{\rho} \mathcal{I}_{0^{+}}^{2-\alpha} u\left(0^{+}\right)}{\Gamma(\alpha-1)} t^{\rho(\alpha-2)} .
\end{aligned}
$$

Finally, for $1<\alpha \leq 2$, we have:

$$
{ }^{\rho} \mathcal{I}_{0^{+}}^{\alpha}{ }^{\rho} \mathcal{D}_{0^{+}}^{\alpha} u(t)=u(t)-\frac{\rho^{1-\alpha}{ }^{\rho} \mathcal{I}_{0^{+}}^{1-\alpha} u\left(0^{+}\right)}{\Gamma(\alpha)} t^{\rho(\alpha-1)}-\frac{\rho^{2-\alpha}{ }^{\rho} \mathcal{I}_{0^{+}}^{2-\alpha} u\left(0^{+}\right)}{\Gamma(\alpha-1)} t^{\rho(\alpha-2)}
$$

As

$$
{ }^{\rho} \mathcal{I}_{0^{+}}^{\alpha} t^{\mu}=\frac{\rho^{-\alpha} \Gamma\left(1+\frac{\mu}{\rho}\right)}{\Gamma\left(1+\alpha+\frac{\mu}{\rho}\right)} t^{\mu+\alpha \rho}, \forall \mu>-\rho
$$


we use $(3.2),(3.3)$, to prove that

$$
\begin{aligned}
& { }^{\rho} \mathcal{I}_{0^{+}}^{1-\alpha}\left[C_{1} t^{\rho(\alpha-1)}\right]=C_{1} \frac{\rho^{-(1-\alpha)} \Gamma\left(1+\frac{\rho(\alpha-1)}{\rho}\right)}{\Gamma\left(1+(1-\alpha)+\frac{\rho(\alpha-1)}{\rho}\right)} t^{\rho(\alpha-1)+(1-\alpha) \rho}=C_{1} \rho^{\alpha-1} \Gamma(\alpha), \\
& { }^{\rho} \mathcal{I}_{0^{+}}^{1-\alpha}\left[C_{2} t^{\rho(\alpha-2)}\right]=C_{2}{ }^{\rho} \mathcal{D}_{0^{+}}^{\alpha-1} t^{\rho(\alpha-2)}=C_{2}{ }^{\rho} \mathcal{D}_{0^{+}}^{\alpha-1} t^{\rho((\alpha-1)-1)}=0
\end{aligned}
$$

for some $C_{1}, C_{2} \in \mathbb{R}$, and

$$
\begin{gathered}
{ }^{\rho} \mathcal{I}_{0^{+}}^{2-\alpha}\left[C_{1} t^{\rho(\alpha-1)}\right]=C_{1} \frac{\rho^{-(2-\alpha)} \Gamma\left(1+\frac{\rho(\alpha-1)}{\rho}\right)}{\Gamma\left(1+(2-\alpha)+\frac{\rho(\alpha-1)}{\rho}\right)} t^{\rho(\alpha-1)+(2-\alpha) \rho}=C_{1} \rho^{\alpha-2} \Gamma(\alpha) t^{\rho} \\
{ }^{\rho} \mathcal{I}_{0^{+}}^{2-\alpha}\left[C_{2} t^{\rho(\alpha-2)}\right]=C_{2} \frac{\rho^{-(2-\alpha)} \Gamma\left(1+\frac{\rho(\alpha-2)}{\rho}\right)}{\Gamma\left(1+(2-\alpha)+\frac{\rho(\alpha-2)}{\rho}\right)} t^{\rho(\alpha-2)+(2-\alpha) \rho}=C_{2} \rho^{\alpha-2} \Gamma(\alpha-1) .
\end{gathered}
$$

Then, for $u(t)=C_{1} t^{\rho(\alpha-1)}+C_{2} t^{\rho(\alpha-2)}$, we have respectively:

$$
\begin{gathered}
{ }^{\rho} \mathcal{I}_{0^{+}}^{1-\alpha} u\left(0^{+}\right)={ }^{\rho} \mathcal{I}_{0^{+}}^{1-\alpha}\left[C_{1} t^{\rho(\alpha-1)}\right]\left(0^{+}\right)+{ }^{\rho} \mathcal{I}_{0^{+}}^{1-\alpha}\left[C_{2} t^{\rho(\alpha-2)}\right]\left(0^{+}\right)=C_{1} \rho^{\alpha-1} \Gamma(\alpha), \\
{ }^{\rho} \mathcal{I}_{0^{+}}^{2-\alpha} u\left(0^{+}\right)={ }^{\rho} \mathcal{I}_{0^{+}}^{2-\alpha}\left[C_{1} t^{\rho(\alpha-1)}\right]\left(0^{+}\right)+{ }^{\rho} \mathcal{I}_{0^{+}}^{2-\alpha}\left[C_{2} t^{\rho(\alpha-2)}\right]\left(0^{+}\right)=C_{2} \rho^{\alpha-2} \Gamma(\alpha-1) .
\end{gathered}
$$

From $(3.4),(3.5),(3.6),(3.7),(3.8),(3.9)$ and (3.10) we get (3.1).

In the following lemma, we define the integral solution of the boundary value problem (1.1)-(1.2) .

Lemma 3.2. Let $\alpha, \rho \in \mathbb{R}^{+}$, be such that $1<\alpha \leq 2$. We give ${ }^{\rho} \mathcal{D}_{0^{+}}^{\alpha} u \in C[0, T]$, and $f(t, u)$ is a continuous function. Then the boundary value problem (1.1)-(1.2), is equivalent to the fractional integral equation

$$
u(t)=\beta \int_{0}^{T} G(t, s) f(s, u(s)) d s, t \in[0, T],
$$

where

$$
G(t, s)= \begin{cases}\frac{\rho^{1-\alpha} s^{\rho-1}}{\Gamma(\alpha)}\left[\left[\frac{t^{\rho}}{T^{\rho}}\left(T^{\rho}-s^{\rho}\right)\right]^{\alpha-1}-\left(t^{\rho}-s^{\rho}\right)^{\alpha-1}\right], & 0 \leq s \leq t \leq T \\ \frac{\rho^{1-\alpha} s^{\rho-1}}{\Gamma(\alpha)}\left[\frac{t^{\rho}}{T^{\rho}}\left(T^{\rho}-s^{\rho}\right)\right]^{\alpha-1}, & 0 \leq t \leq s \leq T\end{cases}
$$

is the Green's function associated with the boundary value problem (1.1)-(1.2).

Proof. Let $\alpha, \rho \in \mathbb{R}^{+}$, be such that $1<\alpha \leq 2$. We apply Lemma 3.1 to reduce the fractional equation (1.1) to an equivalent fractional integral equation. It is easy to 
prove the operator ${ }^{\rho} \mathcal{I}_{0^{+}}^{\alpha}$ has the linearity property for all $\alpha>0$ after direct integration. Then by applying ${ }^{\rho} \mathcal{I}_{0^{+}}^{\alpha}$ to equation (1.1), we get

$$
{ }^{\rho} \mathcal{I}_{0^{+}}^{\alpha}{ }^{\rho} \mathcal{D}_{0^{+}}^{\alpha} u(t)+\beta^{\rho} \mathcal{I}_{0^{+}}^{\alpha} f(t, u(t))=0 .
$$

From Lemma 3.1, we find for $1<\alpha \leq 2$,

$$
{ }^{\rho} \mathcal{I}_{0^{+}}^{\alpha}{ }^{\rho} \mathcal{D}_{0^{+}}^{\alpha} u(t)=u(t)+C_{1} t^{\rho(\alpha-1)}+C_{2} t^{\rho(\alpha-2)},
$$

for some $C_{1}, C_{2} \in \mathbb{R}$. Then, the integral solution of the equation (1.1) is:

$$
u(t)=-\frac{\beta \rho^{1-\alpha}}{\Gamma(\alpha)} \int_{0}^{t} \frac{s^{\rho-1} f(s, u(s))}{\left(t^{\rho}-s^{\rho}\right)^{1-\alpha}} d s-C_{1} t^{\rho(\alpha-1)}-C_{2} t^{\rho(\alpha-2)} .
$$

The conditions (1.2) imply that:

$$
\left\{\begin{array}{lll}
u(0)=0=0-0-\lim _{t \rightarrow 0} C_{2} t^{\rho(\alpha-2)} & \Rightarrow & C_{2}=0, \\
u(T)=0=-\frac{\beta \rho^{1-\alpha}}{\Gamma(\alpha)} \int_{0}^{T} \frac{s^{\rho-1} f(s, u(s))}{\left(T^{\rho}-s^{\rho}\right)^{1-\alpha}} d s-C_{1} T^{\rho(\alpha-1)} & \Rightarrow & C_{1}=-\frac{\beta \rho^{1-\alpha}}{T^{\rho(\alpha-1)} \Gamma(\alpha)} \int_{0}^{T} \frac{s^{\rho-1} f(s, u(s))}{\left(T^{\rho}-s^{\rho}\right)^{1-\alpha}} d s .
\end{array}\right.
$$

The integral equation (3.12) is equivalent to:

$$
u(t)=-\frac{\beta \rho^{1-\alpha}}{\Gamma(\alpha)} \int_{0}^{t} \frac{s^{\rho-1} f(s, u(s))}{\left(t^{\rho}-s^{\rho}\right)^{1-\alpha}} d s+\frac{\beta t^{\rho(\alpha-1)} \rho^{1-\alpha}}{T^{\rho(\alpha-1)} \Gamma(\alpha)} \int_{0}^{T} \frac{s^{\rho-1} f(s, u(s))}{\left(T^{\rho}-s^{\rho}\right)^{1-\alpha}} d s .
$$

Therefore, the unique solution of problem (1.1)-(1.2) is:

$$
\begin{aligned}
u(t)= & \beta \int_{0}^{t} \frac{\rho^{1-\alpha} s^{\rho-1}\left[\left[\frac{t^{\rho}}{T^{\rho}}\left(T^{\rho}-s^{\rho}\right)\right]^{\alpha-1}-\left(t^{\rho}-s^{\rho}\right)^{\alpha-1}\right]}{\Gamma(\alpha)} f(s, u(s)) d s \\
& +\beta \int_{t}^{T} \frac{\rho^{1-\alpha} s^{\rho-1}\left[\frac{t^{\rho}}{T^{\rho}}\left(T^{\rho}-s^{\rho}\right)\right]^{\alpha-1}}{\Gamma(\alpha)} f(s, u(s)) d s \\
= & \beta \int_{0}^{T} G(t, s) f(s, u(s)) d s .
\end{aligned}
$$

The proof is complete.

\subsection{Application of Guo-Krasnosel'skii fixed point theorem}

In this part, we assume that $\beta>0$ and $0<\rho \leq 1$. We impose some conditions on $f$, which allow us to obtain some results on existence of positive solutions for the boundary value problem (1.1)-(1.2) .

We note that $u(t)$ is a solution of (1.1)-(1.2) if and only if:

$$
u(t)=\beta \int_{0}^{T} G(t, s) f(s, u(s)) d s, t \in[0, T] .
$$

Now we prove some properties of the Green's function $G(t, s)$ given by $(3.11)$. 
Lemma 3.3. Let $1<\alpha \leq 2$ and $0<\rho \leq 1$, then the Green's function $G(t, s)$ given by (3.11) satisfies:

(1) $G(t, s)>0$ for $t, s \in(0, T)$.

(2) $\max _{0 \leq t \leq T} G(t, s)=G(s, s)$, for each $s \in[0, T]$.

(3) For any $t \in[0, T]$,

$$
G(t, s) \geq b(t) G(s, s), \text { for any } \frac{T}{8} \leq s \leq T \text { and some } b \in C[0, T] .
$$

Proof. (1) Let $1<\alpha \leq 2$ and $0<\rho \leq 1$. In the case $0<t \leq s<T$, we have:

$$
\frac{\rho^{1-\alpha} s^{\rho-1}}{\Gamma(\alpha)}\left[\frac{t^{\rho}}{T^{\rho}}\left(T^{\rho}-s^{\rho}\right)\right]^{\alpha-1}>0 \text {. }
$$

Moreover, for $0<s \leq t<T$, we have $\frac{t^{\rho}}{T^{\rho}}<1$, then $\frac{t^{\rho}}{T^{\rho}} s^{\rho}<s^{\rho}$ and $t^{\rho}-\frac{t^{\rho}}{T^{\rho}} s^{\rho}>t^{\rho}-s^{\rho}$, thus

$$
t^{\rho}-\frac{t^{\rho}}{T^{\rho}} s^{\rho}=\frac{t^{\rho}}{T^{\rho}}\left(T^{\rho}-s^{\rho}\right)>t^{\rho}-s^{\rho} \Rightarrow\left[\frac{t^{\rho}}{T^{\rho}}\left(T^{\rho}-s^{\rho}\right)\right]^{\alpha-1}-\left(t^{\rho}-s^{\rho}\right)^{\alpha-1}>0,
$$

which imply that $G(t, s)>0$ for any $t, s \in(0, T)$.

(2) To prove that

$$
\max _{0 \leq t \leq T} G(t, s)=G(s, s)=\frac{\rho^{1-\alpha} s^{\rho-1}}{\Gamma(\alpha)}\left[\frac{s^{\rho}}{T^{\rho}}\left(T^{\rho}-s^{\rho}\right)\right]^{\alpha-1}, \forall s \in[0, T],
$$

we choose

$$
\begin{gathered}
g_{1}(t, s)=\frac{\rho^{1-\alpha} s^{\rho-1}}{\Gamma(\alpha)}\left[\left[\frac{t^{\rho}}{T^{\rho}}\left(T^{\rho}-s^{\rho}\right)\right]^{\alpha-1}-\left(t^{\rho}-s^{\rho}\right)^{\alpha-1}\right], \\
g_{2}(t, s)=\frac{\rho^{1-\alpha} s^{\rho-1}}{\Gamma(\alpha)}\left[\frac{t^{\rho}}{T^{\rho}}\left(T^{\rho}-s^{\rho}\right)\right]^{\alpha-1} .
\end{gathered}
$$

Indeed, we put $\max _{0 \leq t \leq T} G(t, s)=G\left(t^{*}, s\right)$, where $0 \leq t^{*} \leq T$. Then, we get for some $0<t_{1}<t_{2}<T$, that

$$
\begin{aligned}
\max _{0 \leq t \leq T} G(t, s) & = \begin{cases}g_{1}\left(t^{*}, s\right), & s \in\left[0, t_{1}\right], \\
\max \left\{g_{1}\left(t^{*}, s\right), g_{2}\left(t^{*}, s\right)\right\}, & s \in\left[t_{1}, t_{2}\right], \\
g_{2}\left(t^{*}, s\right), & s \in\left[t_{2}, T\right],\end{cases} \\
& = \begin{cases}g_{1}\left(t^{*}, s\right), & s \in[0, r], \\
g_{2}\left(t^{*}, s\right), & s \in[r, T],\end{cases}
\end{aligned}
$$

where $r \in\left[t_{1}, t_{2}\right]$, is the unique solution of equation

$$
g_{1}\left(t^{*}, s\right)=g_{2}\left(t^{*}, s\right) \Leftrightarrow t^{*}=s,
$$


which shows the equality (3.14) .

(3) In the following, we divide the proof into two-part, to show the existence $b \in C[0, T]$, such that

$$
G(t, s) \geq b(t) G(s, s), \text { for any } \frac{T}{8} \leq s \leq T
$$

(i) Firstly, if $0 \leq t \leq s \leq T$, we see that $\frac{G(t, s)}{G(s, s)}$ is decreasing with respect to $s$. Consequently

$$
\frac{G(t, s)}{G(s, s)}=\frac{\left[\frac{t^{\rho}}{T^{\rho}}\left(T^{\rho}-s^{\rho}\right)\right]^{\alpha-1}}{\left[\frac{s^{\rho}}{T^{\rho}}\left(T^{\rho}-s^{\rho}\right)\right]^{\alpha-1}}=\left(\frac{t}{s}\right)^{\rho(\alpha-1)} \geq\left(\frac{t}{T}\right)^{\rho(\alpha-1)}=b_{1}(t), \forall t \in[0, s] .
$$

(ii) In the same way, if $0 \leq s \leq t \leq T$, we have $\frac{s^{\rho}}{T^{\rho}}<\frac{t^{\rho}}{T^{\rho}} \leq 1,\left(\frac{t^{\rho}}{T^{\rho}}\right)^{\alpha-2} \geq 1$, $\forall \alpha \in(1,2]$, and

$$
\begin{aligned}
G(t, s) & =\frac{\rho^{1-\alpha} s^{\rho-1}}{\Gamma(\alpha)}\left[\left[\frac{t^{\rho}}{T^{\rho}}\left(T^{\rho}-s^{\rho}\right)\right]^{\alpha-1}-\left(t^{\rho}-s^{\rho}\right)^{\alpha-1}\right] \\
& =\frac{(\alpha-1) \rho^{1-\alpha} s^{\rho-1}}{\Gamma(\alpha)} \int_{t^{\rho}-s^{\rho}}^{\frac{t^{\rho}}{T^{\rho}}\left(T^{\rho}-s^{\rho}\right)} \tau^{\alpha-2} d \tau \\
& \geq \frac{(\alpha-1) \rho^{1-\alpha} s^{\rho-1}}{\Gamma(\alpha)}\left(\frac{t^{\rho}}{T^{\rho}}\right)^{\alpha-2}\left(T^{\rho}-s^{\rho}\right)^{\alpha-2}\left(\frac{t^{\rho}}{T^{\rho}}\left(T^{\rho}-s^{\rho}\right)-\left(t^{\rho}-s^{\rho}\right)\right) \\
& \geq \frac{(\alpha-1) \rho^{1-\alpha} s^{\rho-1}}{\Gamma(\alpha)}\left(T^{\rho}-s^{\rho}\right)^{\alpha-1} \frac{s^{\rho}\left(T^{\rho}-t^{\rho}\right)}{T^{\rho}\left(T^{\rho}-s^{\rho}\right)} .
\end{aligned}
$$

As $0<\rho \leq 1$, we get

$T^{\rho}-t^{\rho}=\rho \int_{t}^{T} \tau^{\rho-1} d \tau \geq \rho T^{\rho-1}(T-t)$, and $T^{\rho}-s^{\rho}=\rho \int_{s}^{T} \tau^{\rho-1} d \tau \leq \rho s^{\rho-1}(T-s)$.

Therefore

$$
\begin{aligned}
\frac{G(t, s)}{G(s, s)} & \geq \frac{\frac{(\alpha-1) \rho^{1-\alpha} s^{\rho-1}}{\Gamma(\alpha)}\left(T^{\rho}-s^{\rho}\right)^{\alpha-1} \frac{s^{\rho}\left(T^{\rho}-t^{\rho}\right)}{T^{\rho}\left(T^{\rho}-s^{\rho}\right)}}{\frac{\rho^{1-\alpha} s^{\rho-1}}{\Gamma(\alpha)}\left[\frac{s^{\rho}}{T^{\rho}}\left(T^{\rho}-s^{\rho}\right)\right]^{\alpha-1}}=(\alpha-1) \frac{s^{\rho}\left(T^{\rho}-t^{\rho}\right)}{T^{\rho}\left(T^{\rho}-s^{\rho}\right)}\left(\frac{T^{\rho}}{s^{\rho}}\right)^{\alpha-1} \\
& \geq(\alpha-1) \frac{s(T-t)}{T(T-s)} \\
& \geq(\alpha-1) \frac{s(T-t)}{T^{2}} .
\end{aligned}
$$

Finally, for $s \in\left[\frac{T}{8}, t\right]$, we have:

$$
\frac{G(t, s)}{G(s, s)} \geq \frac{(\alpha-1)(T-t)}{8 T}=b_{2}(t)
$$


It is clear that $b_{1}(t)$ and $b_{2}(t)$ are positive functions, it is enough to choose:

$$
b(t)= \begin{cases}\left(\frac{t}{T}\right)^{\rho(\alpha-1)}, & \text { for } t \in[0, \bar{t}] \\ \frac{(\alpha-1)(T-t)}{8 T}, & \text { for } t \in[\bar{t}, T]\end{cases}
$$

where $\bar{t} \in(0, T)$ is the unique solution of the equation $b_{1}(t)=b_{2}(t)$. We see that

$$
b(t) \leq \bar{b}=b(\bar{t})=\left(\frac{\bar{t}}{T}\right)^{\rho(\alpha-1)}=\frac{(\alpha-1)(T-\bar{t})}{8 T}<1 \text { for all } t \in[0, T] .
$$

Finally, we have $\forall s \in\left[\frac{T}{8}, T\right]$,

$$
G(t, s) \geq b(t) G(s, s), \forall t \in[0, T] .
$$

The proof is complete.

Lemma 3.4. Let $1<\alpha \leq 2$ and $0<\rho \leq 1$, then there exists a positive constant

$$
\lambda=1+\frac{8^{\rho \alpha} L(\alpha+1)\left[8^{\rho \alpha}-\left(8^{\rho}-1\right)^{\alpha}\right]}{h\left(8^{\rho}-1\right)^{\alpha}\left[8^{\rho}(\alpha+1)+8^{\rho(\alpha-1)}(\alpha-1)\left(8^{\rho}-1\right)\right]} \text {, for some } h, L>0,
$$

such that

$$
\int_{0}^{T} G(s, s) f(s, u(s)) d s \leq \lambda \int_{\frac{T}{8}}^{T} G(s, s) f(s, u(s)) d s .
$$

Proof. As $f(t, u(t)) \geq h$, for any $t \in[0, T]$, we get

$$
\begin{aligned}
\int_{\frac{T}{8}}^{T} G(s, s) f(s, u(s)) d s & \geq h \int_{\frac{T}{8}}^{T} \frac{\rho^{1-\alpha} s^{\rho-1}}{\Gamma(\alpha)}\left[\frac{s^{\rho}}{T^{\rho}}\left(T^{\rho}-s^{\rho}\right)\right]^{\alpha-1} d s \\
& \geq-\frac{h}{\alpha \rho^{\alpha} T^{\rho(\alpha-1)} \Gamma(\alpha)} \int_{\frac{T}{8}}^{T} s^{\rho(\alpha-1)}\left[-\rho \alpha s^{\rho-1}\left(T^{\rho}-s^{\rho}\right)^{\alpha-1}\right] d s .
\end{aligned}
$$

The integral by part gives:

$$
\begin{aligned}
\int_{\frac{T}{8}}^{T} G(s, s) f(s, u(s)) d s & \geq \frac{h\left[\frac{T^{\rho(\alpha-1)}}{8^{\rho(\alpha-1)}}\left(T^{\rho}-\frac{T^{\rho}}{8^{\rho}}\right)^{\alpha}+\rho(\alpha-1) \int_{\frac{T}{8}}^{T} s^{\rho(\alpha-1)-1}\left(T^{\rho}-s^{\rho}\right)^{\alpha} d s\right]}{\rho^{\alpha} T^{\rho(\alpha-1)} \Gamma(\alpha+1)} \\
& \geq \frac{h\left[\frac{T^{\rho}}{8^{\rho(\alpha-1)}}\left(T^{\rho}-\frac{T^{\rho}}{8^{\rho}}\right)^{\alpha}+\rho(\alpha-1) \int_{\frac{T}{8}}^{T} \frac{s^{\rho(\alpha-2)}}{T^{\rho(\alpha-2)}} s^{\rho-1}\left(T^{\rho}-s^{\rho}\right)^{\alpha} d s\right]}{\rho^{\alpha} T^{\rho} \Gamma(\alpha+1)} \\
& \geq \frac{h\left[\frac{T^{\rho}}{8^{\rho(\alpha-1)}}\left(T^{\rho}-\frac{T^{\rho}}{8^{\rho}}\right)^{\alpha}-\frac{\alpha-1}{\alpha+1} \int_{\frac{T}{8}}^{T}\left[-\rho(\alpha+1) s^{\rho-1}\left(T^{\rho}-s^{\rho}\right)^{\alpha}\right] d s\right]}{\rho^{\alpha} T^{\rho} \Gamma(\alpha+1)} \\
& \geq \frac{h T^{\rho \alpha}\left(8^{\rho}-1\right)^{\alpha}}{\rho^{\alpha} 8^{\rho \alpha} \Gamma(\alpha+1)}\left[\frac{8^{\rho}(\alpha+1)+8^{\rho(\alpha-1)}(\alpha-1)\left(8^{\rho}-1\right)}{8^{\rho \alpha}(\alpha+1)}\right] .
\end{aligned}
$$


Then

$$
\frac{\rho^{\alpha} 8^{\rho \alpha} \Gamma(\alpha+1)}{h T^{\rho \alpha}\left(8^{\rho}-1\right)^{\alpha}}\left[\frac{8^{\rho \alpha}(\alpha+1)}{8^{\rho}(\alpha+1)+8^{\rho(\alpha-1)}(\alpha-1)\left(8^{\rho}-1\right)}\right] \int_{\frac{T}{8}}^{T} G(s, s) f(s, u(s)) d s \geq 1 .
$$

On the other hand, if $\max _{0 \leq t \leq T} f(t, u)$ is bounded for $u \in[0, \infty)$, then there exists $L_{0}>0$, such that

$$
|f(t, u(t))| \leq L_{0}, \forall t \in[0, T] .
$$

In the similar way, if $\max _{0 \leq t \leq T} f(t, u)$ is unbounded for $u \in[0, \infty)$, then there exists $M_{0}>0$, such that

$$
\sup _{0 \leq u \leq M_{0}} \max _{0 \leq t \leq T}|f(t, u(t))| \leq L_{1} \text {, for some } L_{1}>0 .
$$

In all cases, for $L=\max \left\{L_{0}, L_{1}\right\}$, we have:

$$
\int_{0}^{\frac{T}{8}} G(s, s) f(s, u(s)) d s \leq L \int_{0}^{\frac{T}{8}} G(s, s) d s \leq \frac{L T^{\rho \alpha}\left[8^{\rho \alpha}-\left(8^{\rho}-1\right)^{\alpha}\right]}{8^{\rho \alpha} \rho^{\alpha} \Gamma(\alpha+1)} .
$$

From (3.17), we get

$$
\begin{aligned}
\int_{0}^{T} G(s, s) f(s, u(s)) d s= & \int_{\frac{T}{8}}^{T} G(s, s) f(s, u(s)) d s+\int_{0}^{\frac{T}{8}} G(s, s) f(s, u(s)) d s \\
\leq & \int_{\frac{T}{8}}^{T} G(s, s) f(s, u(s)) d s+\frac{L T^{\rho \alpha}\left[8^{\rho \alpha}-\left(8^{\rho}-1\right)^{\alpha}\right]}{\rho^{\alpha} 8^{\rho \alpha} \Gamma(\alpha+1)} \\
\leq & \int_{\frac{T}{8}}^{T} G(s, s) f(s, u(s)) d s \\
& +\frac{L T^{\rho \alpha}\left[8^{\rho \alpha}-\left(8^{\rho}-1\right)^{\alpha}\right]}{\rho^{\alpha} 8^{\rho \alpha} \Gamma(\alpha+1)} \times \frac{\rho^{\alpha} 8^{\rho \alpha} \Gamma(\alpha+1)}{h T^{\rho \alpha}\left(8^{\rho}-1\right)^{\alpha}} \\
& \times\left[\frac{8^{\rho \alpha}(\alpha+1)}{8^{\rho}(\alpha+1)+8^{\rho(\alpha-1)}(\alpha-1)\left(8^{\rho}-1\right)}\right] \\
& \times \int_{\frac{T}{8}}^{T} G(s, s) f(s, u(s)) d s \\
\leq & \lambda \int_{\frac{T}{8}}^{T} G(s, s) f(s, u(s)) d s .
\end{aligned}
$$

Let us define the cone $P$ by:

$$
P=\left\{u \in C[0, T] \mid u(t) \geq \frac{b(t)}{\lambda}\|u\|, \forall t \in[0, T]\right\} .
$$


Lemma 3.5. Let $\mathcal{A}: P \rightarrow C[0, T]$ be an integral operator defined by:

$$
\mathcal{A} u(t)=\beta \int_{0}^{T} G(t, s) f(s, u(s)) d s,
$$

equipped with standard norm

$$
\|\mathcal{A} u\|=\max _{0 \leq t \leq T}|\mathcal{A} u(t)|
$$

Then $\mathcal{A}(P) \subset P$.

Proof. For any $u \in P$, we have from $(3.13),(3.16)$ and (3.18), that

$$
\begin{aligned}
\mathcal{A} u(t) & =\beta \int_{0}^{T} G(t, s) f(s, u(s)) d s \geq \beta b(t) \int_{\frac{T}{8}}^{T} G(s, s) f(s, u(s)) d s \\
& \geq \frac{\beta b(t)}{\lambda} \int_{0}^{T} G(s, s) f(s, u(s)) d s \\
& \geq \frac{b(t)}{\lambda} \max _{0 \leq t \leq T}\left(\beta \int_{0}^{T} G(t, s) f(s, u(s)) d s\right) \\
& \geq \frac{b(t)}{\lambda}\|\mathcal{A} u\|, \forall t \in[0, T] .
\end{aligned}
$$

Thus $\mathcal{A}(P) \subset P$. The proof is complete.

Lemma 3.6. $\mathcal{A}: P \rightarrow P$ is a completely continuous operator.

Proof. In view of continuity of $G(t, s)$ and $f(t, u)$, the operator $\mathcal{A}: P \rightarrow P$ is a continuous.

Let $\Omega \subset P$ be a bounded. Then there exists a positive constant $M>0$, such that:

$$
\|u\| \leq M, \forall u \in \Omega
$$

By choice

$$
L=\sup _{0 \leq u \leq M} \max _{0 \leq t \leq T}|f(t, u)|+1 .
$$

In this case, we get $\forall u \in \Omega$,

$$
\begin{aligned}
|\mathcal{A} u(t)| & =\left|\beta \int_{0}^{T} G(t, s) f(s, u(s)) d s\right| \leq \beta \int_{0}^{T}|G(t, s) f(s, u(s))| d s \\
& \leq \beta L \int_{0}^{T} G(s, s) d s \leq \frac{\beta L}{\rho^{\alpha-1} \Gamma(\alpha)} \int_{0}^{T} s^{\rho-1}\left(T^{\rho}-s^{\rho}\right)^{\alpha-1} d s \\
& \leq \frac{\beta L T^{\alpha \rho}}{\rho^{\alpha} \Gamma(\alpha+1)} .
\end{aligned}
$$


Consequently, $|\mathcal{A} u(t)| \leq \frac{\beta L T^{\alpha \rho}}{\rho^{\alpha} \Gamma(\alpha+1)}, \forall u \in \Omega$. Hence, $\mathcal{A}(\Omega)$ is bounded.

Now, for $1<\alpha \leq 2$ and $0<\rho \leq 1$, we give:

$$
\delta(\varepsilon)=\left(\frac{\rho^{\alpha} \Gamma(\alpha)}{T^{\rho} \beta L} \varepsilon\right)^{\frac{1}{\rho(\alpha-1)}}, \text { for some } \varepsilon>0 .
$$

Then $\forall u \in \Omega$, and $t_{1}, t_{2} \in[0, T]$, where $t_{1}<t_{2}$, and $t_{2}-t_{1}<\delta$, we find $\left|\mathcal{A} u\left(t_{2}\right)-\mathcal{A} u\left(t_{1}\right)\right|<\varepsilon$.

Consequently, for $0 \leq s \leq t_{1}<t_{2} \leq T$, we have:

$$
\begin{aligned}
G\left(t_{2}, s\right)-G\left(t_{1}, s\right)= & \frac{\rho^{1-\alpha} s^{\rho-1}}{\Gamma(\alpha)}\left[\left[t_{2}^{\rho(\alpha-1)}-t_{1}^{\rho(\alpha-1)}\right]\left(\frac{T^{\rho}-s^{\rho}}{T^{\rho}}\right)^{\alpha-1}\right. \\
& \left.-\left[\left(t_{2}^{\rho}-s^{\rho}\right)^{\alpha-1}-\left(t_{1}^{\rho}-s^{\rho}\right)^{\alpha-1}\right]\right] \\
& <\frac{\rho^{1-\alpha} s^{\rho-1}}{\Gamma(\alpha)}\left[t_{2}^{\rho(\alpha-1)}-t_{1}^{\rho(\alpha-1)}\right]\left(\frac{T^{\rho}-s^{\rho}}{T^{\rho}}\right)^{\alpha-1} \\
& <\frac{\rho^{1-\alpha} s^{\rho-1}}{\Gamma(\alpha)}\left[t_{2}^{\rho(\alpha-1)}-t_{1}^{\rho(\alpha-1)}\right] .
\end{aligned}
$$

In the same way, for $0 \leq t_{1} \leq s<t_{2} \leq T$ or $0 \leq t_{1}<t_{2} \leq s \leq T$, we have:

$$
G\left(t_{2}, s\right)-G\left(t_{1}, s\right)<\frac{\rho^{1-\alpha} s^{\rho-1}}{\Gamma(\alpha)}\left[t_{2}^{\rho(\alpha-1)}-t_{1}^{\rho(\alpha-1)}\right] .
$$

Then

$$
\begin{aligned}
\left|\mathcal{A} u\left(t_{2}\right)-\mathcal{A} u\left(t_{1}\right)\right| & =\left|\beta \int_{0}^{T}\left[G\left(t_{2}, s\right)-G\left(t_{1}, s\right)\right] f(s, u(s)) d s\right| \\
& \leq \beta L \int_{0}^{T}\left|G\left(t_{2}, s\right)-G\left(t_{1}, s\right)\right| d s \\
& <\beta L \int_{0}^{T} \frac{\rho^{1-\alpha} s^{\rho-1}}{\Gamma(\alpha)}\left[t_{2}^{\rho(\alpha-1)}-t_{1}^{\rho(\alpha-1)}\right] d s \\
& <\frac{\beta L \rho^{1-\alpha}}{\Gamma(\alpha)}\left[t_{2}^{\rho(\alpha-1)}-t_{1}^{\rho(\alpha-1)}\right]\left[\frac{1}{\rho} s^{\rho}\right]_{0}^{T}
\end{aligned}
$$

Finally

$$
\left|\mathcal{A} u\left(t_{2}\right)-\mathcal{A} u\left(t_{1}\right)\right|<\frac{\beta L T^{\rho}}{\rho^{\alpha} \Gamma(\alpha)}\left[t_{2}^{\rho(\alpha-1)}-t_{1}^{\rho(\alpha-1)}\right] .
$$

In the following, we divide the proof into three cases.

(a) If $\delta \leq t_{1}<t_{2} \leq T$, we have:

$$
\delta \leq t_{1}<t_{2} \Leftrightarrow t_{2}^{\rho(\alpha-2)}<t_{1}^{\rho(\alpha-2)} \leq \delta^{\rho(\alpha-2)}, \text { and } t_{2}^{\rho-1}<t_{1}^{\rho-1} \leq \delta^{\rho-1} .
$$

Thus

$$
t_{2}^{\rho}-t_{1}^{\rho}=t_{2} t_{2}^{\rho-1}-t_{1} t_{1}^{\rho-1}<t_{2} t_{2}^{\rho-1}-t_{1} t_{2}^{\rho-1}=t_{2}^{\rho-1}\left(t_{2}-t_{1}\right)<\delta^{\rho-1}\left(t_{2}-t_{1}\right)<\delta^{\rho} .
$$


In similar way

$$
\begin{aligned}
t_{2}^{\rho(\alpha-1)}-t_{1}^{\rho(\alpha-1)} & =t_{2}^{\rho} t_{2}^{\rho(\alpha-2)}-t_{1}^{\rho} t_{1}^{\rho(\alpha-2)}<t_{2}^{\rho} t_{2}^{\rho(\alpha-2)}-t_{1}^{\rho} t_{2}^{\rho(\alpha-2)}=t_{2}^{\rho(\alpha-2)}\left(t_{2}^{\rho}-t_{1}^{\rho}\right) \\
& <\delta^{\rho(\alpha-2)}\left(t_{2}^{\rho}-t_{1}^{\rho}\right) \\
& <\delta^{\rho(\alpha-1)} .
\end{aligned}
$$

Then, the inequality (3.20) gives:

$$
\begin{aligned}
\left|\mathcal{A} u\left(t_{2}\right)-\mathcal{A} u\left(t_{1}\right)\right| & <\frac{\beta L T^{\rho}}{\rho^{\alpha} \Gamma(\alpha)}\left[t_{2}^{\rho(\alpha-1)}-t_{1}^{\rho(\alpha-1)}\right]<\frac{\beta L T^{\rho}}{\rho^{\alpha} \Gamma(\alpha)} \delta^{\rho(\alpha-1)} \\
& <\frac{\beta L T^{\rho}}{\rho^{\alpha} \Gamma(\alpha)}\left[\left(\frac{\rho^{\alpha} \Gamma(\alpha)}{T^{\rho} \beta L} \varepsilon\right)^{\frac{1}{\rho(\alpha-1)}}\right]^{\rho(\alpha-1)} \\
& <\varepsilon .
\end{aligned}
$$

(b) If $t_{1} \leq \delta<t_{2}<2 \delta$, we have:

$$
t_{1} \leq \delta<t_{2} \Leftrightarrow t_{2}^{\rho(\alpha-2)}<\delta^{\rho(\alpha-2)} \leq t_{1}^{\rho(\alpha-2)},
$$

and

$$
\begin{aligned}
t_{2}^{\rho(\alpha-1)}-t_{1}^{\rho(\alpha-1)} & =t_{2}^{\rho} t_{2}^{\rho(\alpha-2)}-t_{1}^{\rho} t_{1}^{\rho(\alpha-2)}<t_{2}^{\rho} \delta^{\rho(\alpha-2)}-t_{1}^{\rho} \delta^{\rho(\alpha-2)} \\
& <\delta^{\rho(\alpha-2)}\left(t_{2}^{\rho}-t_{1}^{\rho}\right)<\delta^{\rho(\alpha-1)}
\end{aligned}
$$

Also, we find the same result (3.21).

(c) If $t_{1}<t_{2} \leq \delta$, we have:

$$
\begin{aligned}
\left|\mathcal{A} u\left(t_{2}\right)-\mathcal{A} u\left(t_{1}\right)\right| & <\frac{\beta L T^{\rho}}{\rho^{\alpha} \Gamma(\alpha)}\left[t_{2}^{\rho(\alpha-1)}-t_{1}^{\rho(\alpha-1)}\right]<\frac{\beta L T^{\rho}}{\rho^{\alpha} \Gamma(\alpha)} t_{2}^{\rho(\alpha-1)} \\
& <\frac{\beta L T^{\rho}}{\rho^{\alpha} \Gamma(\alpha)} \delta^{\rho(\alpha-1)} \\
& <\varepsilon .
\end{aligned}
$$

By the means of the Ascoli-Arzel Theorem 2.11, we have $\mathcal{A}: P \rightarrow P$ is completely continuous.

We define some important constants

$$
\begin{array}{ll}
F_{0}=\lim _{u \rightarrow 0^{+}} \max _{t \in[0, T]} \frac{f(t, u)}{u}, & F_{\infty}=\lim _{u \rightarrow+\infty} \max _{t \in[0, T]} \frac{f(t, u)}{u}, \\
f_{0}=\lim _{u \rightarrow 0^{+}} \min _{t \in[0, T]} \frac{f(t, u)}{u}, & f_{\infty}=\lim _{u \rightarrow+\infty} \min _{t \in[0, T]} \frac{f(t, u)}{u}, \\
\omega_{1}=\int_{0}^{T} G(s, s) d s, & \omega_{2}=\frac{\bar{b}}{\lambda^{2}} \int_{0}^{T} G(s, s) b(s) d s .
\end{array}
$$

Assume that $\frac{1}{\omega_{2} f_{\infty}}=0$ if $f_{\infty} \rightarrow \infty, \frac{1}{\omega_{1} F_{0}}=\infty$ if $F_{0} \rightarrow 0, \frac{1}{\omega_{2} f_{0}}=0$ if $f_{0} \rightarrow \infty$, and $\frac{1}{\omega_{1} F_{\infty}}=\infty$ if $F_{\infty} \rightarrow 0$. 
Theorem 3.7. If $\omega_{2} f_{\infty}>\omega_{1} F_{0}$ holds, then for each:

$$
\beta \in\left(\left(\omega_{2} f_{\infty}\right)^{-1},\left(\omega_{1} F_{0}\right)^{-1}\right),
$$

the boundary value problem (1.1)-(1.2) has at least one positive solution.

Proof. Let $\beta$ satisfies (3.22) and $\varepsilon>0$, be such that

$$
\left(\left(f_{\infty}-\varepsilon\right) \omega_{2}\right)^{-1} \leq \beta \leq\left(\left(F_{0}+\varepsilon\right) \omega_{1}\right)^{-1} .
$$

From the definition of $F_{0}$, we see that there exists $r_{1}>0$, such that

$$
f(t, u) \leq\left(F_{0}+\varepsilon\right) u, \forall t \in[0, T], 0<u \leq r_{1} .
$$

Consequently, for $u \in P$ with $\|u\|=r_{1}$, we have from $(3.23),(3.24)$, that

$$
\begin{aligned}
\|\mathcal{A} u\| & =\max _{0<t<T}\left|\beta \int_{0}^{T} G(t, s) f(s, u(s)) d s\right| \\
& \leq \beta \int_{0}^{T} G(s, s)\left(F_{0}+\varepsilon\right) u(s) d s \\
& \leq \beta\left(F_{0}+\varepsilon\right)\|u\| \int_{0}^{T} G(s, s) d s \\
& \leq \beta\left(F_{0}+\varepsilon\right)\|u\| \omega_{1} \\
& \leq\|u\| .
\end{aligned}
$$

Hence, if we choose $\Omega_{1}=\left\{u \in C[0, T]:\|u\|<r_{1}\right\}$, then

$$
\|\mathcal{A} u\| \leq\|u\|, \text { for } u \in P \cap \partial \Omega_{1} .
$$

By definition of $f_{\infty}$, there exists $r_{3}>0$, such that

$$
f(t, u) \geq\left(f_{\infty}-\varepsilon\right) u, \forall t \in[0, T], u \geq r_{3} .
$$

Therefore, for $u \in P$ with $\|u\|=r_{2}=\max \left\{2 r_{1}, r_{3}\right\}$, we have from $(3.23),(3.26)$, that

$$
\begin{aligned}
\|\mathcal{A} u\| & \geq \mathcal{A} u(\bar{t})=\beta \int_{0}^{T} G(\bar{t}, s) f(s, u(s)) d s \geq \beta \int_{\frac{T}{8}}^{T} b(\bar{t}) G(s, s) f(s, u(s)) d s \\
& \geq \frac{\beta \bar{b}}{\lambda} \int_{0}^{T} G(s, s) f(s, u(s)) d s \geq \frac{\beta \bar{b}}{\lambda} \int_{0}^{T} G(s, s)\left[\left(f_{\infty}-\varepsilon\right) u(s)\right] d s, \quad \forall t \in[0, T] .
\end{aligned}
$$

By definition of $P$ in (3.18), we have:

$$
\begin{aligned}
\|\mathcal{A} u\| & \geq \frac{\beta \bar{b}\left(f_{\infty}-\varepsilon\right)}{\lambda^{2}}\|u\| \int_{0}^{T} G(s, s) b(s) d s \\
& \geq \beta\left(f_{\infty}-\varepsilon\right)\|u\| \omega_{2} \\
& \geq\|u\| .
\end{aligned}
$$


If we set $\Omega_{2}=\left\{u \in C[0, T]:\|u\|<r_{2}\right\}$, then

$$
\|\mathcal{A} u\| \geq\|u\|, \text { for } u \in P \cap \partial \Omega_{2} .
$$

Now, from $(3.25),(3.27)$, and Lemma 2.13, we guarantee that $\mathcal{A}$ has a fix point $u \in P \cap\left(\bar{\Omega}_{2} \backslash \Omega_{1}\right)$ with $r_{1} \leq\|u\| \leq r_{2}$. It is clear that $u$ is a positive solution of (1.1)-(1.2). The proof is complete.

Theorem 3.8. If $\omega_{2} f_{0}>\omega_{1} F_{\infty}$ holds, then for each:

$$
\beta \in\left(\left(\omega_{2} f_{0}\right)^{-1},\left(\omega_{1} F_{\infty}\right)^{-1}\right),
$$

the boundary value problem (1.1)-(1.2) has at least one positive solution.

Proof. Let $\beta$ satisfies (3.28) and $\varepsilon>0$, be such that

$$
\left(\left(f_{0}-\varepsilon\right) \omega_{2}\right)^{-1} \leq \beta \leq\left(\left(F_{\infty}+\varepsilon\right) \omega_{1}\right)^{-1} .
$$

From definition of $f_{0}$, we see that there exists $r_{1}>0$, such that

$$
f(t, u) \geq\left(f_{0}-\varepsilon\right) u, \forall t \in[0, T], 0<u \leq r_{1} .
$$

Further, if $u \in P$ with $\|u\|=r_{1}$, then similar to the proof's second part of Theorem 3.7, we can get that $\|\mathcal{A} u\| \geq\|u\|$. Then, if we choose $\Omega_{1}=\left\{u \in C[0, T]:\|u\|<r_{1}\right\}$, thus

$$
\|\mathcal{A} u\| \geq\|u\|, \text { for } u \in P \cap \partial \Omega_{1} .
$$

Next, and by definition of $F_{\infty}$, we may choose $R_{1}>0$, such that

$$
f(t, u) \leq\left(F_{\infty}+\varepsilon\right) u, \text { for } u \geq R_{1} .
$$

We consider two cases:

1) If $\max _{0 \leq t \leq T} f(t, u)$ is bounded for $u \in[0, \infty)$. Then, there exists some $L>0$, such that

$$
f(t, u) \leq L, \text { for all } t \in[0, T], u \in P .
$$

Let us denote by $r_{3}=\max \left\{2 r_{1}, \beta L \omega_{1}\right\}$, if $u \in P$ with $\|u\|=r_{3}$, then

$$
\|\mathcal{A} u\|=\max _{0 \leq t \leq T}\left|\beta \int_{0}^{T} G(t, s) f(s, u(s)) d s\right| \leq \beta L \int_{0}^{T} G(s, s) d s=\beta L \omega_{1} \leq r_{3}=\|u\| .
$$

Hence,

$$
\|\mathcal{A} u\| \leq\|u\|, \text { for } u \in \partial P_{r_{3}}=\left\{u \in P:\|u\| \leq r_{3}\right\} .
$$

2) If $\max _{0 \leq t \leq T} f(t, u)$ is unbounded for $u \in[0, \infty)$, then there exists some $r_{4}=$ $\max \left\{2 r_{1}, R_{1}\right\}$, such that

$$
f(t, u) \leq \max _{0 \leq t \leq T} f\left(t, r_{4}\right), \text { for all } 0<u \leq r_{4}, t \in[0, T] .
$$


Let $u \in P$ with $\|u\|=r_{4}$. Then, from (3.29), (3.31), we have:

$$
\begin{aligned}
\|\mathcal{A} u\| & =\max _{0<t<T}\left|\beta \int_{0}^{T} G(t, s) f(s, u(s)) d s\right| \leq \beta \int_{0}^{T} G(s, s)\left(F_{\infty}+\varepsilon\right) u(s) d s \\
& \leq \beta\left(F_{\infty}+\varepsilon\right)\|u\| \int_{0}^{T} G(s, s) d s=\beta\left(F_{\infty}+\varepsilon\right)\|u\| \omega_{1} \\
& \leq\|u\| .
\end{aligned}
$$

Thus, (3.32) is also true for $u \in \partial P_{r_{4}}$.

In both cases 1 and 2 , if we set $\Omega_{2}=\left\{u \in C[0, T]:\|u\|<r_{2}=\max \left\{r_{3}, r_{4}\right\}\right\}$, then

$$
\|\mathcal{A} u\| \leq\|u\|, \text { for } u \in P \cap \partial \Omega_{2} .
$$

Now, from $(3.30),(3.33)$, and Lemma 2.13, we guarantee that $\mathcal{A}$ has a fix point $u \in P \cap\left(\bar{\Omega}_{2} \backslash \Omega_{1}\right)$ with $r_{1} \leq\|u\| \leq r_{2}$. It is clear that $u$ is a positive solution of (1.1)-(1.2). The proof is complete.

Theorem 3.9. Suppose there exists $r_{2}>r_{1}>0$, such that

$$
\sup _{0 \leq u \leq r_{2}} \max _{0 \leq t \leq T} f(t, u) \leq \frac{r_{2}}{\beta \omega_{1}}, \text { and } \inf _{0 \leq u \leq r_{1}} f(t, u) \geq \frac{r_{1}}{\beta \lambda \omega_{2}} b(t), \forall t \in[0, T] .
$$

Then, the boundary value problem (1.1)-(1.2) has a positive solution $u \in P$, with $r_{1} \leq\|u\| \leq r_{2}$.

Proof. Choose $\Omega_{1}=\left\{u \in C[0, T]:\|u\|<r_{1}\right\}$. Then, for $u \in P \cap \partial \Omega_{1}$, we get

$$
\begin{aligned}
\|\mathcal{A} u\| & \geq \mathcal{A} u(\bar{t})=\beta \int_{0}^{T} G(\bar{t}, s) f(s, u(s)) d s \geq \beta \int_{\frac{T}{8}}^{T} b(\bar{t}) G(s, s) f(s, u(s)) d s \\
& \geq \frac{\beta \bar{b}}{\lambda} \int_{0}^{T} G(s, s) \inf _{0 \leq u \leq r_{1}} f(s, u(s)) d s \geq \frac{\beta \bar{b}}{\lambda} \int_{0}^{T} G(s, s) \frac{r_{1}}{\beta \lambda \omega_{2}} b(s) d s \\
& \geq r_{1}=\|u\| .
\end{aligned}
$$

On the other hand, choose $\Omega_{2}=\left\{u \in C[0, T]:\|u\|<r_{2}\right\}$. Then, for $u \in P \cap \partial \Omega_{2}$, we get

$$
\begin{aligned}
\|\mathcal{A} u\| & =\max _{0<t<T}\left|\beta \int_{0}^{T} G(t, s) f(s, u(s)) d s\right| \leq \beta \int_{0}^{T} G(s, s) \sup _{0 \leq u \leq r_{2}} \max _{0 \leq t \leq T} f(s, u(s)) d s \\
& \leq \beta \int_{0}^{T} G(s, s) \frac{r_{2}}{\beta \omega_{1}} d s=r_{2}=\|u\| .
\end{aligned}
$$

Now, from Lemma 2.13 , we guarantee that $\mathcal{A}$ has a fix point $u \in P \cap\left(\bar{\Omega}_{2} \backslash \Omega_{1}\right)$ with $r_{1} \leq\|u\| \leq r_{2}$. It is clear that $u$ is a positive solution of (1.1)-(1.2). The proof is complete. 


\subsection{Application of Banach fixed point theorem}

In this part, we assume that $\beta \in \mathbb{R}$ and $\rho>0$, and $f:[0, T] \times[0, \infty) \rightarrow[0, \infty)$ satisfies the conditions:

(H1) $f(t, u)$ is Lebesgue measurable function with respect to $t$ on $[0, T]$,

(H2) $f(t, u)$ is continuous function with respect to $u$ on $\mathbb{R}$.

Theorem 3.10. Assume (H1), (H2) hold, and there exists a constant $\sigma>0$, such that

$$
|f(t, u)-f(t, v)| \leq \sigma|u-v|, \text { for almost every } t \in[0, T] \text {, and all } u, v \in C[0, T] .
$$

If

$$
|\beta|<\frac{\rho^{\alpha} \Gamma(\alpha+1)}{\sigma T^{\alpha \rho}} .
$$

Then, there exists a unique solution of the boundary value problem (1.1)-(1.2) on $[0, T]$.

Proof. Assume that $|\beta|<\frac{\rho^{\alpha} \Gamma(\alpha+1)}{\sigma T^{\alpha \rho}}$, and consider the operator $\mathcal{A}: C[0, T] \rightarrow C[0, T]$ defined by (3.19) as follows

$$
\mathcal{A} u(t)=\beta \int_{0}^{T} G(t, s) f(s, u(s)) d s .
$$

We shall show that $\mathcal{A}$ is a contraction mapping. In fact, for any $u, v \in C[0, T]$, we have

$$
\begin{aligned}
|\mathcal{A} u(t)-\mathcal{A} v(t)| & =\left|\beta \int_{0}^{T} G(t, s)[f(s, u(s))-f(s, v(s))] d s\right| \\
& \leq|\beta| \int_{0}^{T} G(t, s)|f(s, u(s))-f(s, v(s))| d s \\
& \leq|\beta| \sigma \int_{0}^{T} G(s, s)|u(s)-v(s)| d s,
\end{aligned}
$$

then

$$
\begin{aligned}
\|\mathcal{A} u-\mathcal{A} v\| & \leq|\beta| \sigma\|u-v\| \int_{0}^{T} G(s, s) d s \\
& \leq \frac{|\beta| \sigma T^{\alpha \rho}}{\rho^{\alpha} \Gamma(\alpha+1)}\|u-v\| .
\end{aligned}
$$

This imply from (3.37) that $\mathcal{A}$ is a contraction operator. As a consequence of Theorem 2.14 , by Banach's contraction principle [5], we deduce that $\mathcal{A}$ has a unique fixed point which is the unique solution of the problem (1.1)-(1.2) on $[0, T]$. 


\section{Examples}

In this section, we present some examples to illustrate the usefulness of our main results.

Example 1. Consider the following boundary value problem

$$
\left\{\begin{array}{l}
{ }^{1} \mathcal{D}_{0^{+}}^{\frac{3}{2}} u(t)+\beta(1+t) u(t) \ln (1+u(t))=0, \quad t \in[0,1] \\
u(0)=u(1)=0
\end{array}\right.
$$

Set $\beta>0$ any finite positive real number, and

$$
f(t, u)=(1+t) u \ln (1+u) .
$$

In this case, the function $f$ is jointly continuous for any $t \in[0,1]$, and any $u>0$.

We get

$$
F_{0}=\lim _{u \rightarrow 0^{+}} \max _{t \in[0, T]} \frac{f(t, u)}{u}=0^{+}, \quad f_{\infty}=\lim _{u \rightarrow+\infty} \min _{t \in[0, T]} \frac{f(t, u)}{u}=\infty
$$

On the other hand, we get

$$
\omega_{1}=\int_{0}^{1} G(s, s) d s=\frac{1}{\Gamma\left(\frac{3}{2}\right)} \int_{0}^{1} \sqrt{s(1-s)} d s=\frac{1}{\frac{1}{2} \sqrt{\pi}} \frac{\pi}{8}=\frac{\sqrt{\pi}}{4},
$$

and

$$
b(t)= \begin{cases}\sqrt{t} & \text { for } t \in[0, \bar{t}] \\ \frac{1-t}{16} & \text { for } t \in[\bar{t}, 1]\end{cases}
$$

Then

$$
\omega_{2}=\frac{\bar{b}}{\lambda^{2} \Gamma\left(\frac{3}{2}\right)}\left[\int_{0}^{\bar{t}} s \sqrt{(1-s)} d s+\frac{1}{16} \int_{\bar{t}}^{1} \sqrt{s}(1-s)^{\frac{3}{2}} d s\right] \simeq \frac{\bar{b} \sqrt{\pi}}{128 \lambda^{2}}
$$

Where $\bar{t} \simeq 0,003876 \ldots$ and $\bar{b} \simeq 0,062258 \ldots$ and the choice of $\lambda$ depends directly by choice of $r_{1}, r_{2}$ in $(3.25),(3.27)$.

Because $\omega_{1}, \omega_{2}>0$, two finite constants for any choice of $0<r_{1}<r_{2}<\infty$. We have always:

$$
\frac{1}{\omega_{2} f_{\infty}}=0, \text { and } \frac{1}{\omega_{1} F_{0}}=\infty
$$

Then, the condition (3.22) is satisfied for any $0<\beta<\infty$.

It follows from Theorem 3.7 that the problem (4.1) has at least one solution.

Example 2. Consider

$$
\left\{\begin{array}{l}
{ }^{1} \mathcal{D}_{0^{+}}^{\frac{3}{2}} u(t)+\beta(1+t) u(t) \exp \left(\frac{1}{u(t)}-[u(t)]^{2}\right)=0, \quad t \in[0,1] . \\
u(0)=u(1)=0 .
\end{array}\right.
$$


Set $\beta>0$ any finite positive real number, and

$$
f(t, u)=(1+t) u \exp \left(\frac{1}{u}-u^{2}\right) .
$$

Clearly, for any $t \in[0,1]$ and any $u>0$, the function $f$ is jointly continuous.

Here, we have:

$$
f_{0}=\lim _{u \rightarrow 0^{+}} \min _{t \in[0, T]} \frac{f(t, u)}{u}=\infty, \quad F_{\infty}=\lim _{u \rightarrow+\infty} \max _{t \in[0, T]} \frac{f(t, u)}{u}=0^{+}
$$

Also, we find the same function $b(t)$ in $(4.3)$, and same constant $\omega_{1}, \omega_{2}$ respectively in $(4.2),(4.4)$.

The choice of $\lambda>1$ depends directly by choice of $r_{1}, r_{2}$ in $(3.30),(3.33)$.

Because $\omega_{1}, \omega_{2}>0$, two finite constants for any choice of $0<r_{1}<r_{2}<\infty$. We have always:

$$
\frac{1}{\omega_{2} f_{0}}=0, \text { and } \frac{1}{\omega_{1} F_{\infty}}=\infty .
$$

Then, the condition (3.28) is satisfied for any $0<\beta<\infty$.

It follows from Theorem 3.8 that the problem (4.5) has at least one solution.

Example 3. Consider the following boundary value problem

$$
\left\{\begin{array}{l}
{ }^{1} \mathcal{D}_{0^{+}}^{\frac{3}{2}} u(t)+\frac{(1+t)(1+u(t))}{\sqrt{\pi}}=0, \quad t \in[0,1] . \\
u(0)=u(1)=0 .
\end{array}\right.
$$

Set $\beta=\frac{1}{\sqrt{\pi}}$, and

$$
f(t, u)=(1+t)(1+u) .
$$

The function $f$ is jointly continuous for any $t \in[0,1]$ and any $u>0$.

We find the same function $b(t)$ in $(4.3)$, such that $0 \leq b(t)<1$, and

$$
\omega_{1}=\int_{0}^{1} G(s, s) d s=\frac{\sqrt{\pi}}{4} .
$$

Choosing $r_{1}=\frac{1}{10^{4}}<r_{2}=2$. Then, for all $t \in[0,1]$, we have:

$$
h=1 \leq f(t, u) \leq 6=L .
$$

In this case

$$
\begin{aligned}
\lambda & =1+\frac{8^{\rho \alpha} L(\alpha+1)\left[8^{\rho \alpha}-\left(8^{\rho}-1\right)^{\alpha}\right]}{h\left(8^{\rho}-1\right)^{\alpha}\left[8^{\rho}(\alpha+1)+8^{\rho(\alpha-1)}(\alpha-1)\left(8^{\rho}-1\right)\right]} \\
& =1+\frac{8^{\frac{3}{2}} \times 6 \times \frac{5}{2} \times\left(8^{\frac{3}{2}}-7^{\frac{3}{2}}\right)}{7^{\frac{3}{2}} \times\left(8 \times \frac{5}{2}+\sqrt{8} \times \frac{7}{2}\right)} \\
& \simeq 3,517426 \ldots
\end{aligned}
$$


Then

$$
\omega_{2} \simeq \frac{\bar{b} \sqrt{\pi}}{128 \lambda^{2}} \simeq \frac{0,062258 \times \sqrt{\pi}}{128 \times 3,517426^{2}} \simeq \frac{3,9313 \sqrt{\pi}}{10^{5}} .
$$

It remains to show that the conditions in $(3.34)$, which is

$$
\sup _{0 \leq u \leq r_{2}} \max _{0 \leq t \leq T} f(t, u)=6 \leq \frac{r_{2}}{\beta \omega_{1}} \simeq 8
$$

and

$$
\inf _{0 \leq u \leq r_{1}} f_{3}(t, u)=1+t \geq \frac{r_{1}}{\beta \lambda \omega_{2}} b(t) \simeq 0,72317 \times b(t), \quad \forall t \in[0,1] .
$$

Are satisfied. It follows from Theorem 3.9 that the problem (4.6) has at least one solution.

\section{Example 4. Let}

$$
\left\{\begin{array}{l}
{ }^{\frac{2}{3}} \mathcal{D}_{0^{+}}^{\frac{3}{2}} u(t)+\frac{\cos (t)[2+|u(t)|]}{\pi(\sqrt{2} \cos (t)+\sin (t))[1+|u(t)|]}=0, \quad t \in\left[0, \frac{\pi}{4}\right] \\
u(0)=u\left(\frac{\pi}{4}\right)=0
\end{array}\right.
$$

Set $\beta=\frac{1}{\pi}$ and

$$
f(t, u)=\frac{\cos (t)[2+|u|]}{(\sqrt{2} \cos (t)+\sin (t))[1+|u|]}, \quad t \in\left[0, \frac{\pi}{4}\right], u, v \in \mathbb{R}
$$

As $\sin (t), \cos (t)$ are continuous positive functions $\forall t \in\left[0, \frac{\pi}{4}\right]$, the function $f$ is jointly continuous. For any $u, v \in \mathbb{R}$ and $t \in\left[0, \frac{\pi}{4}\right]$, we have $\frac{\sqrt{2}}{2} \leq \cos (t) \leq 1$, and $0 \leq \sin (t) \leq \frac{\sqrt{2}}{2}$, then

$$
\begin{aligned}
|f(t, u)-f(t, v)| & =\left|\frac{\cos (t)[2+|u|]}{(\sqrt{2} \cos (t)+\sin (t))[1+|u|]}-\frac{\cos (t)[2+|v|]}{(\sqrt{2} \cos (t)+\sin (t))[1+|v|]}\right| \\
& =\left|\frac{\cos (t)}{\sqrt{2} \cos (t)+\sin (t)}\right|\left|\frac{2+|u|}{1+|u|}-\frac{2+|v|}{1+|v|}\right| \\
& \leq|| u|-| v|| \leq|u-v| .
\end{aligned}
$$

Hence, the condition (3.35) is satisfied with $\sigma=1$. It remains to show that the condition (3.36)

$$
0<\beta=\frac{1}{\pi} \simeq 0,318309 \ldots<\frac{\rho^{\alpha} \Gamma(\alpha+1)}{\sigma T^{\alpha \rho}}=\frac{\frac{2}{3}^{\frac{3}{2}} \times \Gamma\left(\frac{5}{2}\right)}{\frac{\pi}{4}} \simeq 0,921317 \ldots
$$

is satisfied. It follows from Theorem 3.10 that the problem (4.7) has a unique solution. 


\section{Conclusion}

In this paper we have discussed the existence and the uniqueness of solutions for a class of nonlinear fractional differential equations with a boundary value, by using the properties of Guo-Krasnosel'skii and Banach fixed point theorems. The used differential operator is developed by Katugampola, which generalizes the RiemannLiouville and the Hadamard fractional derivatives into a single form.

\section{Acknowledgments}

The authors are deeply grateful to the referees and the editors for their kind comments on improving the presentation of this paper.

\section{References}

[1] Y. Arioua, N. Benhamidouche, Boundary value problem for Caputo-Hadamard fractional differential equations, Surveys in Mathematics and its Applications 12 (2017) 103-115.

[2] R.P. Agarwal, M. Meehan, D. O'Regan, Fixed Point Theory and Applications, Cambridge University Press, Cambridge, 2001.

[3] K. Diethelm, The Analysis of Fractional Differential Equations, Springer, Berlin, 2010 .

[4] M. El-Shahed, Positive solutions for boundary value problem of nonlinear fractional differential equation, Abstract and Applied Analysis 2007 (2007) 1-8.

[5] A. Granas, J. Dugundji, Fixed Point Theory, Springer-Verlag, New York, 2003.

[6] U.N. Katugampola, New approach to a generalized fractional integral, Applied Mathematics and Computation 218 (3) (2011) 860-865.

[7] U.N. Katugampola, A new approach to generalized fractional derivatives, Mathematical Analysis and Applications 6 (4) (2014) 1-15.

[8] U.N. Katugampola, Existence and uniqueness results for a class of generalized fractional differential equations, Bull. Math. Anal. Appl., arXiv:1411.5229v1 (2016).

[9] A.A. Kilbas, H.H. Srivastava, J.J. Trujillo, Theory and Applications of Fractional Differential Equations, Elsevier Science B.V., Amsterdam, 2006.

[10] A.A. Kilbas, J.J. Trujillo, Differential equations of fractional order: methods, results and problems I, Appl. Anal. 78 (2001) 153-192. 
[11] A.A. Kilbas, J.J. Trujillo, Differential equations of fractional order: methods, results and problems II, Appl. Anal. 81 (2002) 435-493.

[12] M.A. Krasnosel'skii, Positive Solutions of Operator Equations, Noordhoff, Groningen, 1964.

[13] R.W. Leggett, L.R. Williams, Multiple positive fixed points of nonlinear operators on ordered Banach spaces, Indiana Univ. Math. J. 28 (1979) 673-688.

[14] K.S. Miller, Fractional differential equations, J. Fract. Calc. 3 (1993) 49-57.

[15] K.S. Miller, B. Ross, An Introduction to the Fractional Calculus and Fractional Differential Equations, Wiley, New York, 1993.

[16] A.M. Nakhushev, The Sturm-Liouville problem for a second order ordinary differential equation with fractional derivatives in the lower terms, Dokl. Akad. Nauk SSSR 234 (1977) 308-311.

[17] I. Podlubny, Fractional Differential Equations, Mathematics in Science and Engineering, Academic Press, New York, 1999.

[18] S.G. Samko, A.A. Kilbas, O.I. Marichev, Fractional Integral and Derivatives (Theory and Applications), Gordon and Breach, Switzerland, 1993.

[19] X. Xu, D. Jiang, C. Yuan, Multiple positive solutions for the boundary value problem of a nonlinear fractional differential equation, Nonlinear Anal. 71 (2009) 4676-4688.

[20] Zhanbing Bai, Haishen L, Positive solutions for boundary value problem of nonlinear fractional differential equation, J. Math. Anal. Appl. 311 (2005) 495-505.

\section{DOI: $10.7862 / \mathrm{rf.2019.3}$}

\section{Bilal Basti}

email: bilalbasti@univ-msila.dz

ORCID: 0000-0001-8216-3812

Laboratory for Pure and Applied Mathematics

University of M'sila

M'sila 28000

ALGERIA 


\section{Yacine Arioua*}

email: yacine.arioua@univ-msila.dz

ORCID: 0000-0002-9681-9568

Laboratory for Pure and Applied Mathematics

University of M'sila

M'sila 28000

ALGERIA

*Corresponding author

Nouredine Benhamidouche

email: nbenhamidouche@univ-msila.dz

ORCID: 0000-0002-5740-8504

Laboratory for Pure and Applied Mathematics

University of M'sila

M'sila 28000

ALGERIA

Received 03.08.2018

Accepted 29.12.2018 